\title{
FEM ANALYSIS ON MIXED-MODE FRACTURE OF CSM-GRP
}

\author{
Zhang Shuang-yin (张双寅) \\ (Institute of Mechanics, Chinese Academy of Sciences, Beijing) \\ C. M. Leech \\ (Dept. of Mech. Eng., UMIST, U. K.)
}

(Received Mar. 15, 1985 Communicated by Chien Wei-zang)

\begin{abstract}
A FEM analysis for studying mixed-mode fracture problem of chopped strand mat glass fibre reinforced polyester laminate is presented. The analysis is formulated on the basis of 8-node quadrilateral isoparametric element. The collapsed triangular quarter-point singular elements were used for calculating stress intensity factors $K_{I}$ and $K \boldsymbol{I}$.

The crack propagation process was computed by implementing constraint release technique. Three different approaches to the solution of stress intensity factors $K_{I}$ and $K_{I I}$ were compared. The effect of constraint condition imposed upon the displacement of the three collapsed nodes of the crack tip elements on the $K_{I}$ and $K_{I I}$ results was evaluated. The mixed-mode critical stress intensity factors $K_{I C}$ and $K_{I I}$ were estimated for CSM-GRP through the consideration of $K_{1}$ and $K_{I I}$ calculated and the measured failure load and critical crack length in the experiment.
\end{abstract}

\section{Introduction}

Many finite element approaches to crack problems (fracture problems) have been developed ${ }^{\text {(I) }}$ ${ }^{[2]}$. The collapsed triangular quarter-point element degenerated from quadrilateral isoparametric element has now been accepted as one of the best elements of calculating fracture toughness and proved to be able to produce more satisfactory results ${ }^{[3][4]}$.

A numer of ways to estimate the stress intensity factors from finite element displacement near a crack tip were proposed by different investigators ${ }^{[3] \mid[\mid}$. The constraint condition imposed on the three nodes on the collapsed side of the singular element renders a large effect on the strain singularity. Reference [6] has demonstrated that the triangular quarter-point elements could have either $1 / \mathrm{N} r$ singularity or $1 / r$ singularity, that depends on if the three collapsed nodes are constrained to have the same displacement or not. The proof of the different singularity was also given in Reference [7].

While a large number of papers of investigating crack problem in isotropic (metallic) materials have been published, there are not many on the finite element analysis of the crack problem in fibre composites. $\mathrm{Sih}^{[8]}$ has studied fracture mechanics of composite materials and pointed out that in some circumstances the well-established fracture mechanics for metal materials can be applied to investigation of composite material problem. Wang etc. published a paper [9] on mixed-mode crack problem of rectilinear anisotropic solids; as an example, he dealt with the problem of the 
crack emanating from a circular hole in E-glass/epoxy panel.

In the present paper, a FEM analysis for studying the interlaminar shear fracture behaviour of chopped strand mat reinforced polyester laminate is presented. The deformation of the doublegrooved (lap-shear) specimen and stress distributions along the shear surface are calculated using 2 dimensional 8-node quadrilateral elements. The crack initiation point and initial crack propagation direction are predicted.

The micro-cracked (damage) zone is calculated by using iieration method. Three failure criteria are employed in the calculations and the results obtained are compared. In this paper, crackextension process is studied, in which the constraint release technique is utilized. The mixed-mode stress intensity factors $K_{l}$ and $K_{I I}$ from finite element displacement field in the near crack zone are computed.

The effect of the constraint condition imposed upon the three collapsed nodes of the singular elements on the values of $K_{l}$ and $K_{I I}$ is evaluated. The critical stress intensity factors $K_{I C}$ and $K_{I I C}$ are estimated by using $K_{I}$ and $K_{I I}$ calculated and the critical crack length and failure load measured in the experiment.

\section{Finite Element Formulation}

\section{2-D quadratic isoparametric element}

In the present paper two dimensional 8-node isoparametric (Serendipity) quadrilateral element are used. In the isoparametric element, the same shape function is used in both the coordinate representation of $x$ and $y$, and the displacement representation.

a) The coordinate representations can be given as:

$$
X=\sum_{i=1}^{8} N_{i}(\xi, \eta) X_{i}, \quad Y=\sum_{i=1}^{s} N_{i}(\xi, \eta) Y_{i}
$$

and the displacement representations can be expressed as:

$$
U=\sum_{i=1}^{8} N_{i}(\xi, \eta) U_{i}, \quad V=\sum_{i=1}^{8} N_{i}(\xi, \eta) V_{i}
$$

The shape function is:

$$
\begin{aligned}
& N_{1}=\frac{1}{4}\left(1+\xi_{0}\right)\left(1+\eta_{0}\right)\left(\xi_{0}+\eta_{0}-1\right), \quad i=1,3,5,7 \\
& N_{1}=\frac{1}{2}\left(1-\xi^{2}\right)\left(1+\eta_{0}\right), \quad i=2,6 \\
& N_{1}=\frac{1}{2}\left(1-\eta^{2}\right)\left(1+\xi_{0}\right), \quad i=4,8
\end{aligned}
$$

where:

$$
\xi_{0}=\xi_{1} \xi, \eta_{0}=\eta_{i} \eta
$$

and $\xi_{i}$ and $\eta_{i}$ equal +1 or -1 correspondingly.

b) Strain-displacement relationship is given as:

$$
\{\varepsilon\}=\left\{\begin{array}{l}
\varepsilon_{x} \\
\varepsilon_{y} \\
\gamma_{x y}
\end{array}\right\}=[B]\{\delta\}^{*}
$$


where $\{\delta\}^{\bullet}$ expresses the node displacement vector of an element, that is:

$$
\{\delta\}^{e}=\left[u_{1}, v_{1}, u_{2}, v_{2}, \cdots, u_{8}, v_{8}\right]^{r}
$$

and $[B]$ is strain matrix and given as:

$$
\left[B_{i}\right]^{(\bullet)}=\left(\begin{array}{cc}
{\left[\frac{\partial N_{i}}{\partial x}\right]^{(e)}} & 0 \\
0 & {\left[\frac{\partial N_{i}}{\partial y}\right]^{(\bullet)}} \\
{\left[\frac{\partial N_{i}}{\partial y}\right]^{(\bullet)}} & {\left[\frac{\partial N_{i}}{\partial x}\right]^{(\bullet)}}
\end{array}\right)
$$

c) Stress-strain relationship is as follows:

$$
\{\sigma\}=\left\{\begin{array}{c}
\sigma_{x} \\
\sigma_{y} \\
\tau_{x y}
\end{array}\right\}=[D]\{\varepsilon\}
$$

Where $[D]$ is the constitutive matrix and is given as:

$$
[D]=\frac{1}{1-v_{12} v_{21}}\left[\begin{array}{ccc}
E_{11} & v_{21} E_{11} & 0 \\
& E_{22} & 0 \\
\text { Sym } & & G_{12}
\end{array}\right]
$$

for orthotropic materia model.

d) The element nodal force vector is given as:

$$
\{F\}^{(\bullet)}=\{F\}(\because)+\{F\}(\because+\{F\} \stackrel{(\bullet)}{a}
$$

where $\{F\} ;$ is concentrated force vector.

$\{F\}$ is gravitational force vector and can be derived from:

$$
\{F\}^{r}=\int_{0}\left[N_{0}\right]^{T}\{w\} d \Omega
$$

and $\{F\}:$ is distributed force vector, and can be derived from:

$$
\{F\}_{a}^{\prime}=\int_{\Gamma}\left[N_{\mathbf{l}}\right]^{T}\{q\} d s
$$

e) The element stiffness matrix can be derived from the virtual work principle. From the virtual work expression the following equation can be obtained:

$$
\{F\}^{(e)}=[K]^{\cdot}:\{\delta\} \cdot
$$

where $[K]^{\cdot}$ is element stiffness matrix.

$$
[K]^{(e)}=\int_{-1}^{1} \int_{-1}^{1}[B]^{x}[D][B]|J| d \xi d \eta
$$

where $|J|$ is Jacobian determinant,

$$
|J|=\left|\begin{array}{ll}
\frac{\partial x}{\partial \xi} & \frac{\partial y}{\partial \xi} \\
\frac{\partial x}{\partial \eta} & \frac{\partial y}{\partial \eta}
\end{array}\right|
$$




\section{The formulation of special element}

In expressions (2.1) and (2.2) if the midside node is just located at the middle of the sides, within the element coordinate, $r$ (in polar coordinate system) does not have $\sqrt{r}$ term, this element does not have $1 / \sqrt{r}$ singularity. But, if the midside node is placed at the location decided according to the following formula:

$$
r_{2}=\frac{\left(\sqrt{r_{1}}+\sqrt{r_{3}}\right)^{2}}{4}
$$

the element will have $1 / \sqrt{r}$ singularity, and it can be proved that:

$$
\xi=-\frac{\sqrt{r_{1}}+\sqrt{r_{3}}}{\sqrt{r_{3}}-\sqrt{r_{1}}}+\frac{2 \sqrt{r}}{\sqrt{r_{3}}-\sqrt{r_{1}}}
$$

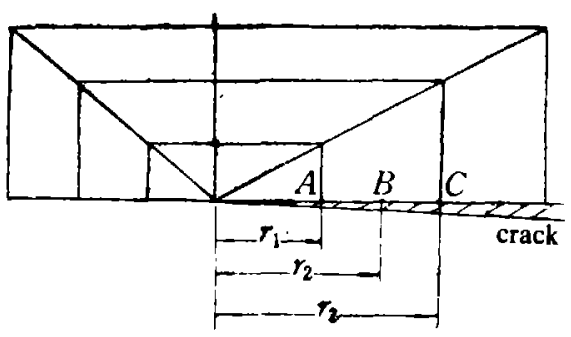

where $r_{1}, r_{2}$ and $r_{3}$ are distances of the three nodes on crack surface from the crack tip (See Fig. 1). When $r_{l}=0$, $r_{2}=r_{3} / 4$, the quarter point element was obtained.

Fig. 1 Special elements

\section{Determlnation Of Stress Intensity Factors}

\section{Isotropic material model}

The classical linear elastic fracture mechanics has the following formulas which express the relationship between the displacement field in the vicinity of the crack tip and the stress intensity factors $K_{I}$ and $K_{I I}$.

$$
\begin{aligned}
& U_{r}=\frac{K_{1}}{4 G}\left(\frac{r}{2 \pi}\right)^{\frac{1}{2}} f_{11}(\theta)-\frac{K_{1}}{4 G}\left(\frac{r}{2 \pi}\right)^{\frac{1}{2}} f_{11}(\theta) \\
& U_{0}=\frac{K_{1}}{4 G}\left(\frac{r}{2 \pi}\right)^{\frac{1}{2}} f_{12}(\theta)-\frac{K_{1}}{4 G}\left(\frac{r}{2 \pi}\right)^{\frac{1}{2}} f_{12}(\theta)
\end{aligned}
$$

where:

$$
\begin{aligned}
& f_{I_{1}}(\theta)=(2 \kappa-1) \cos \frac{\theta}{2}-\cos \frac{3 \theta}{2} \\
& f_{I_{1}}(\theta)=(2 \kappa-1) \sin \frac{\theta}{2}-3 \sin \frac{3 \theta}{2} \\
& f_{I_{2}}(\theta)=\sin \frac{3 \theta}{2}-(1+2 \kappa) \sin \frac{\theta}{2} \\
& f_{I_{2}}(\theta)=(2 \kappa+1) \cos \frac{\theta}{2}-3 \cos \frac{3 \theta}{2}
\end{aligned}
$$

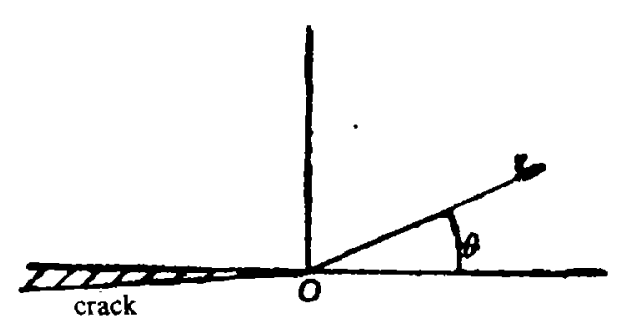

(3.2d) Fig. 2 Polar coordinate in formulas (3.1) and (3.2)

$G$ is shear modulus, $G=E / 2(1+v)$,

$$
\text { and } k= \begin{cases}3-4 v & \text { for plane strain problems } \\ \frac{3-v}{1+v} & \text { for plane stress problems }\end{cases}
$$

The polar coordinate system is shown in Fig.2.

\section{Anisotropic material model}




$$
\begin{aligned}
U_{r}= & K_{1} \sqrt{\frac{2 r}{\pi}} \operatorname{Re}\left[\frac{1}{\mu_{1}-\mu_{2}}\left(\mu_{1} p_{2} \sqrt{\cos \theta+\mu_{2} \sin \theta}-\mu_{2} p_{1} \sqrt{\cos \theta+\mu_{1} \sin \theta}\right)\right] \\
& +K_{1} \sqrt{\frac{2 r}{\pi}} \operatorname{Re}\left[\frac{1}{\mu_{1}-\mu_{2}}\left(p_{2} \sqrt{\cos \theta+\mu_{2} \sin \theta}-p_{1} \sqrt{\cos \theta+\mu_{1} \sin \theta}\right)\right] \\
U_{0}= & K_{1} \sqrt{\frac{2 r}{\pi}} \operatorname{Re}\left[\frac{1}{\mu_{1}-\mu_{2}}\left(\mu_{1} q_{2} \sqrt{\cos \theta+\mu_{2} \sin \theta}-\mu_{2} q_{1} \sqrt{\cos \theta+\mu_{1} \sin \theta}\right)\right] \\
& +K_{1} \sqrt{\frac{12 r}{\pi}} \operatorname{Re}\left[\frac{1}{\mu_{1}-\mu_{2}}\left(q_{2} \sqrt{\cos \theta+\mu_{2} \sin \theta}-q_{1} \sqrt{\cos \theta+\mu_{1} \sin \theta}\right)\right]
\end{aligned}
$$

where $\mu_{1}$ and $\mu_{2}$ are the two different roots of the following equation, and they always occur in conjugate pairs, as $\mu_{1}, \bar{\mu}_{2}$ and $\mu_{2}, \bar{\mu}_{2}$.

$$
a_{11} \mu^{4}-2 a_{18} \mu^{3}+\left(2 a_{12}+a_{68}\right) \mu^{2}-2 a_{28} \mu+a_{22}=0
$$

$p_{i}$ and $q_{i}$ are defined as:

$$
p_{6}=a_{11} \mu_{1}^{2}+a_{12}-a_{10} \mu_{i}, q_{1}=a_{12} \mu_{i}+\frac{a_{22}}{\mu_{6}}-a_{20}
$$

In equation (3.4) and formulas (3.5a) and (3.5b), $a_{i j}$ are compliance coefficients.

$$
\varepsilon_{i}=\sum a_{i j} \sigma_{j}
$$

\section{Determinates of $K_{I}$ and $K_{\text {I }}$ from the near crack displacement field}

Formulas (3.1) and (3.3) express the relationship between displacements $U_{r}$ and $U_{\theta}$ and $K_{I}$ and $K_{I I}$. Using the displacement distribution on the two crack surfaces $(\theta= \pm \pi)$ to obtain $K_{I}$ and $K_{I I}$ is the simplest way and gives more accurate results.

For isotropic material:

$$
\begin{aligned}
& K_{I}(\pi)=\sqrt{\frac{2 \pi}{r_{0}}} \cdot \frac{2 G U_{\theta}\left(r_{0}, \pi\right)}{(\kappa+1)} \quad(\theta=\pi) \\
& K_{1}(-\pi)=\sqrt{\frac{2 \pi}{r_{0}}} \cdot \frac{2 G U_{0}\left(r_{0},-\pi\right)}{(\kappa+1)} \quad(\theta=-\pi) \\
& K_{1}=\frac{K_{1}(\pi)+K_{1}(-\pi)}{2} \text {. } \\
& K_{\mathrm{I}}(\pi)=\sqrt{\frac{2 \pi}{r_{0}}} \cdot \frac{2 G U_{r}\left(r_{0}, \pi\right)}{(\kappa+1)} \quad(\theta=\pi) \\
& K_{1}(-\pi)=\sqrt{\frac{2 \pi}{r_{0}}} \cdot \frac{2 G U_{r}\left(r_{0},-\pi\right)}{(\kappa+1)} \quad(\theta=-\pi) \\
& K_{\mathrm{I}}=\frac{K_{1}(\pi)+K_{1}(-\pi)}{2}
\end{aligned}
$$

For orthotropic material, there are :ormulas similar to (3.7) and (3.8). The only change is factor $(\kappa+1)$, which should be calculated from formulas (3.3) through (3.6), and be different for $K_{1}$ and $K_{I I}$.

It can be seen that $K_{I}$ and $K_{I I}$ obtained from formulas (3.7) through (3.8) depend on the value of $r_{o}$. To obtain $K_{I}$ and $K_{I I}$ at the crack tip, the following three approaches have been used in different 
papers:

(a) Let $r_{0}$ be equal to $1-2$ per cent of the crack length, or $r_{o}=\mathrm{a} / 9 .^{[3] .}$ The values of $K_{I}$ and $K_{I I}$ obtained from formulas (3.7) through (3.8) are good estimation of true $K_{I}$ and $K$

(b) Calculate $K_{I}$ and $K_{I I}$ from the following formulas ${ }^{[5]}$ :

$$
\left.\begin{array}{l}
K_{1}=\frac{2 G \sqrt{2 \pi}}{\kappa+1}\left\{\frac{\left.4 U_{\theta}\right|_{B}-\left.U_{\theta}\right|_{c}}{\sqrt{ } L}\right\} \\
K_{I}=\frac{2 G \sqrt{2 \pi}}{\kappa+1}\left\{\frac{\left.{ }_{4} U_{r}\right|_{B}-\left.U_{r}\right|_{c}}{\sqrt{L}}\right\}
\end{array}\right\}
$$

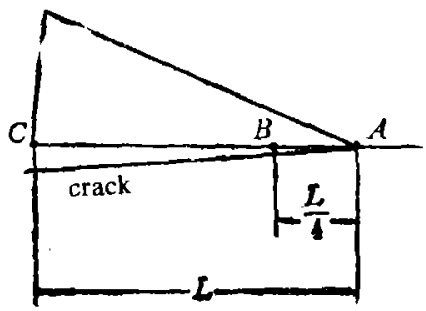

where $\left.U_{\theta}\right|_{B},\left.U_{\theta}\right|_{C},\left.U_{\mathrm{F}}\right|_{B}$, and $\left.U_{+}\right|_{C}$ are circumferential Fig.3 A quarter-point element and radial displacements at points $B$ and $C$ respectively. (See Fig.3)

(c) Extrapolation method

It has been shown in [4] that the $K_{/}$and $K_{/ /}$results obtained from formulas of (3.1 a)through (3.2d) vary linearly with $r$. Select a series of $r_{t}$, calculate $\left(K_{l}\right)$, and $\left(K_{l l}\right)$., then use a straight line extrapolate to obtain $K_{l}$ and $K_{\| /}$at $r=0$.

\section{Singularity of the collapsed triangular quarter-point elements}

The collapsed triangular quarter-point elements could have $1 / \sqrt{ } r$ or $1 / r$ singularity in the strain field near crack tip, depending on whether the three collapsed nodes having the same coordinates of crack tip are constrained to have same displacements or not. If the following constraint conditions are imposed on them,

$$
U_{1}=U_{j}=U_{h}, V_{i}=V_{j}=V_{k}
$$

where $i, j, k$ are node numbers of the collapsed nodes, the strain field obtained in this element will have $1 / \mathrm{N} r$ singularity. This element applies to calculation of linear elastic stress intensity factors, because stress field also has $1 / N r$ singularity. But, if the three nodes can be displaced independently, the strain field calculated will be of $1 / r$ singularity. In this case, the stress field is constant in the vicinity of crack tip, this eiement applies to perfectly plastic crack problems.

\section{Analysis of Interlaminar Shear Fracture of Chopped Strand Mat Glassfibre Reinforced Plestic (CSM-CRP)}

The interlaminar shear fracture behaviour has been studied both experimentally and analytically, the main results will be presented in another paper. The specimen tested is shown in Fig.4, it is a plare strain problem.

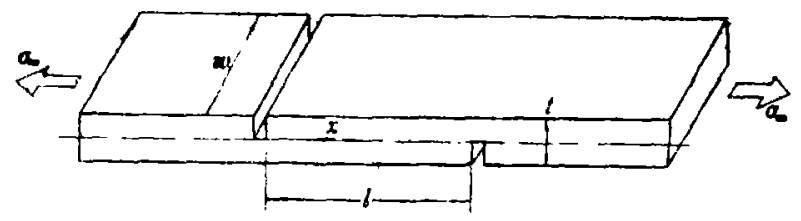

Fig.4 A lap-shear specimen

\section{Precracked deformation and stress distribution in the specimen}

The precrack deformation and stress distribution were computed by using finite element mesh pattern (1), shown in Fig.5, and the results obtatned are shown in Fig.6 to Fig.8. The stress values in 


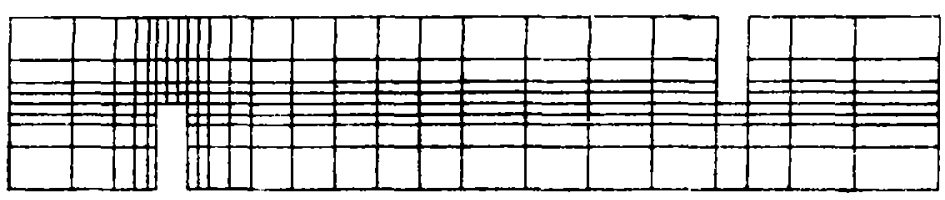

Fig.5 Finite Element mesh pattern (I)

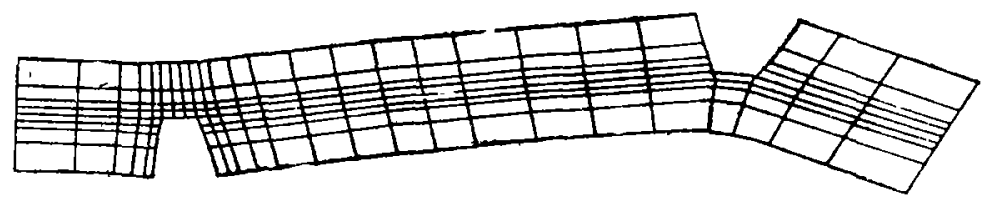

Fig.6 Deformed shape of mesh pattern (I)
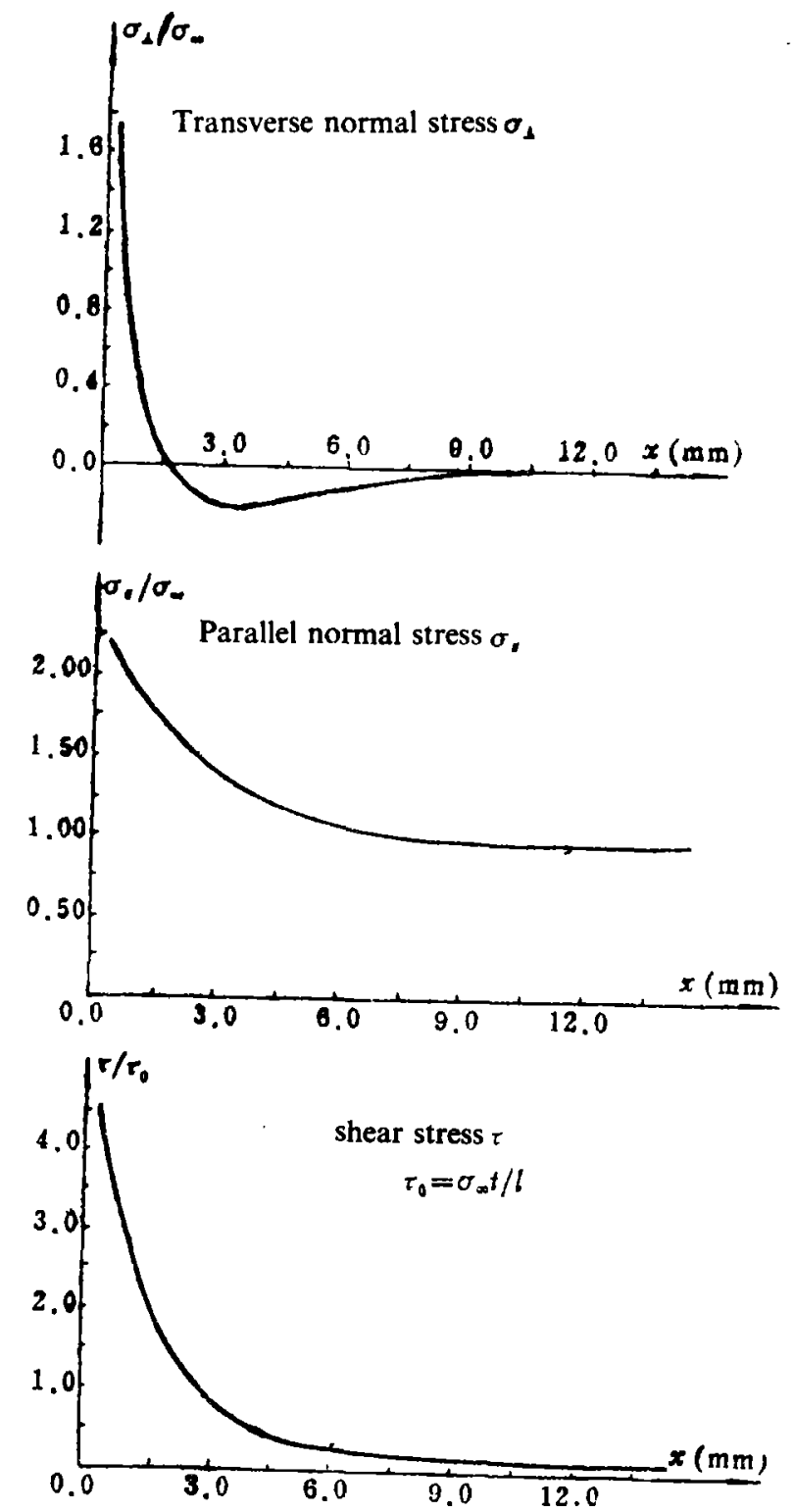

Fig.7 Stress distributions (isotropic material) 
the three figures are those of average stress in elements. Fig. 7 is for isotropic material model, in which $E=7.0 \mathrm{GPa}, \quad \nu=0.34$; and Fig.8 is for orthotropic material model, in which $E_{11}=9.81$ $\mathrm{GPa}, \quad E_{22}=5.20 \mathrm{GPa}, \quad v_{12}=0.34, G_{12}=1.43 \mathrm{GPa}$.
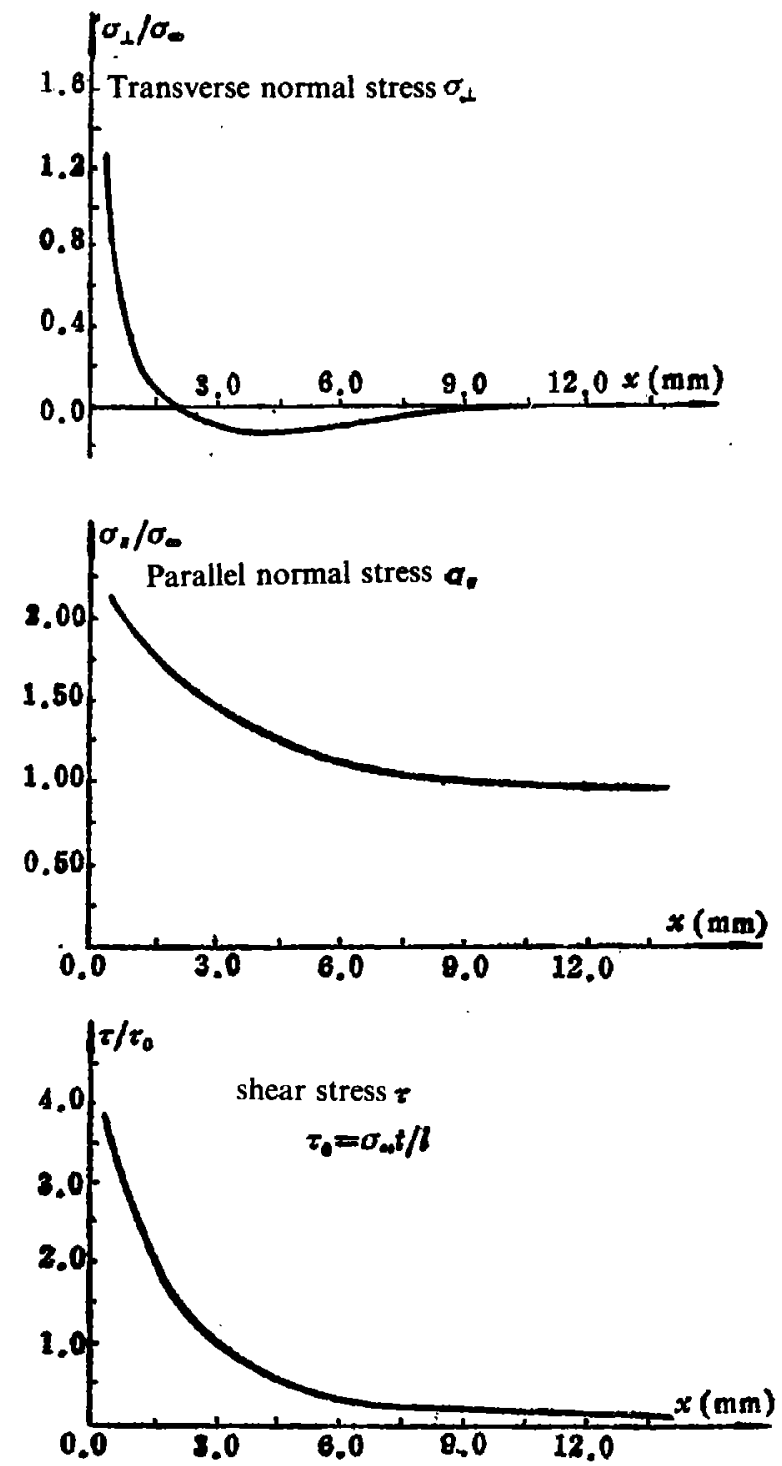

Fig. 8 Stress distributions(orthotropic material)

\section{Calculations of the microcracked (damage) zone.}

The calculations for predicting the crack initiation and microcracked zone extension were carried out by using the mesh shown in Fig. 5 . Within every element the strains and stresses at 25 $(5 \times 5)$ points (including 9 Gauss sampling points and 8 nodal points) can be calculated. In each load step the maximum stress and the maximum stress point were searched. If the stress is larger than a critical value, this point will fail, a microcrack occurs. The following criteria were employed in the computation $^{[10]}$ :

a. Maximum stress failure criteria 


$$
\sigma_{\|} \leqslant \sigma_{u e}, \sigma_{\perp} \leqslant \sigma_{\perp c}, \tau \leqslant \tau_{c} \quad(4.1 \mathrm{a}, \mathrm{b}, \mathrm{c})
$$

b. Norris distortional energy failure criterion

$$
\phi=\sqrt{\left(\frac{\sigma_{n}}{\sigma_{u c}}\right)^{2}-\frac{\sigma_{u} \sigma_{\perp}}{\sigma_{u c} \sigma_{\perp c}}+\left(\frac{\sigma_{\perp}}{\sigma_{\perp c}}\right)^{2}+\left(\frac{\tau}{\tau_{c}}\right)^{2}} \leqslant 1
$$

In formulas (4.1) and (4.2), $\sigma_{n c}, \sigma_{\perp}$ and $\tau_{\odot}$ are critical stresses, and from experimental data available we assume:

$$
\sigma_{11 c}=120 \mathrm{MPa}, \sigma_{\perp c}=9.0 \mathrm{MPa}, \tau_{c}=9.5 \mathrm{MPa}
$$

Making use of the following procedure, the damage zone is calculated:

a) For each load increment the maximum stress (or stress factor $\phi$ ) point is searched.

b) Checking for failure using criteria (4.1) or (4.2).

c) If this point fails, the corresponding stiffness coefficient in constitutive matrix $[D]$ will be set equal to zero. For example:

if $\tau \geqslant \tau_{0}$, then $G_{12}=0$,

if $\sigma_{\perp} \geqslant \sigma_{\perp e}$, then $E_{22}=v_{21}=0$,

if $\sigma_{n} \geqslant \sigma_{n 0}$, then $E_{11}=v_{12}=0$,

if $\phi \geqslant 1$, then $E_{11}=E_{22}=G_{12}=0$.

Matrix $[D]$ at other points and other elements remains unchanged.

d) Modify the stiffness matrix and repeat the same computation procedure described above, damage zone obtained.

The different failure criteria predict the same crack initiation point, that is the inner corner point of the two notch zs. Whereas the initial damage strength predicted by different criterion is different. The results are listed in Table 1.

Table 1

\begin{tabular}{c|c|c|c|c}
\hline Criteria & $\sigma_{y}<\sigma_{n c}$ & $\sigma_{\perp}<\sigma_{\perp_{c}}$ & $\tau<\tau_{c}$ & $\phi<1$ \\
\hline Initial Strength & $20.2 \mathrm{MPa}$ & $2.45 \mathrm{MPa}$ & $3.64 \mathrm{MPa}$ & $2.1 \mathrm{MPa}$ \\
\hline
\end{tabular}

From Table 1 it can be concluded that the distortional strain energy criterion, $\phi \leqslant 1$, predicts an earlier damage than other criteria. The maximum parallel stress criterion, $\sigma_{u} \leqslant \sigma_{u} e$, predicts a much larger strength. It is unlikely for the parallel stress $\sigma_{a}$ to cause the material failure.

The initial crack extension direction can be predicted through the searching for the next (the second) failure point. The three criteria, ie. $\phi \leqslant 1, \sigma_{\perp} \leqslant \sigma_{\perp}$ and $\tau \leqslant \tau_{0}$, all predict the same initial crack extension direction, that is along the interface (shear surface). Because in this calculation at the failure points the material is not broken (separated) completely, but it is still continuous, we name this area damaged (or microcracked) zone. The damaged zone predicted by the criterion $r \leqslant \tau_{0}$ is shown in Fig.9, the numbers indicate the failure sequences. The stress distribution along the shear surface of the specimen with the damage zone calculated by using $\phi \leqslant 1$ criterian is illustrated in Fig.10. The stress distribution curves obtained by the other"two criteria have the same feature. The stress values (average in the element) at the corner of the notch diminish notably. 


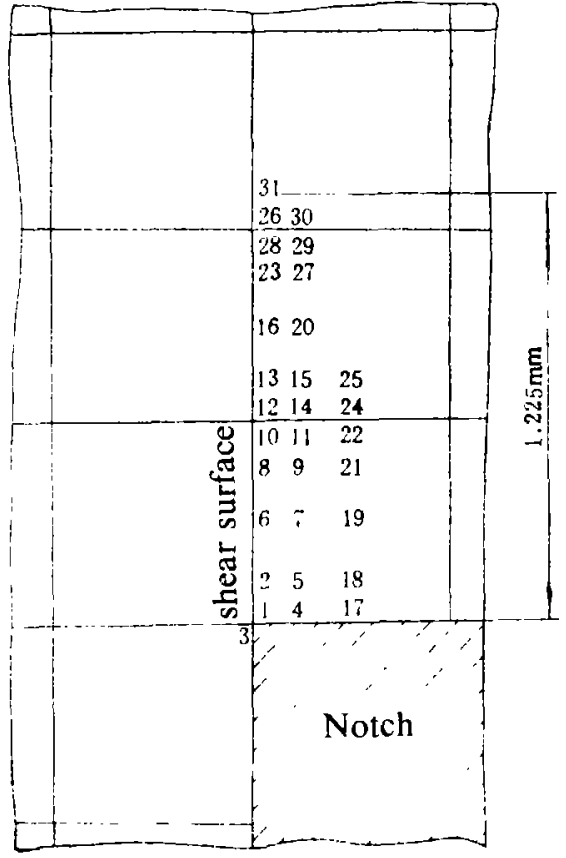

Fig.9 Damaged zone calculated with the criterion of $\tau \leqslant \tau$, (Load= $4.97 \mathrm{MPa})$
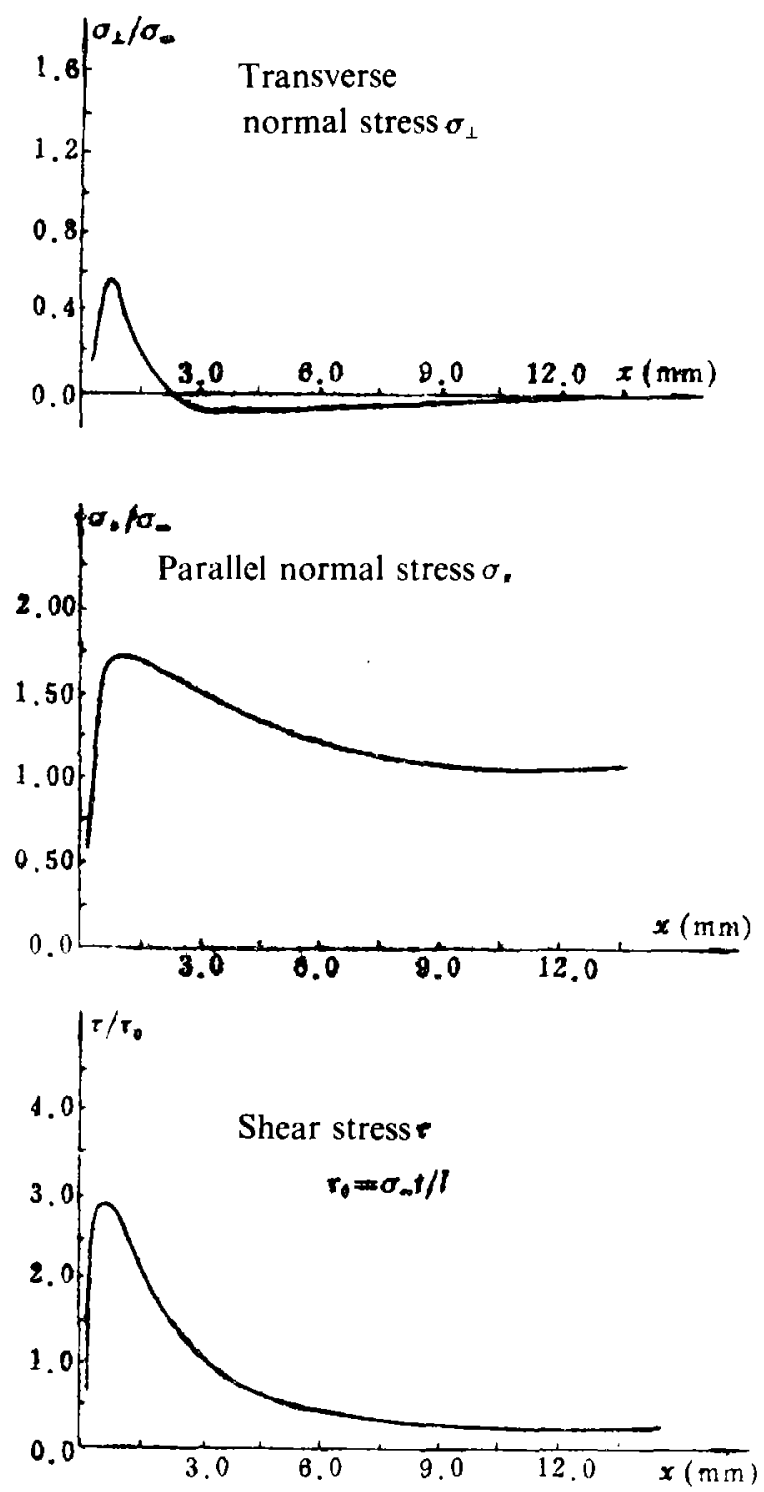

Fig.10 Stress distributions along shear surface with a damaged zone predicted by $\phi \leqslant 1$

\section{Mixed-mode stress intensity factors}

The crack propagation process and the stress intensity factors are calculated by using the finite element mesh pattern II (See Fig.11).

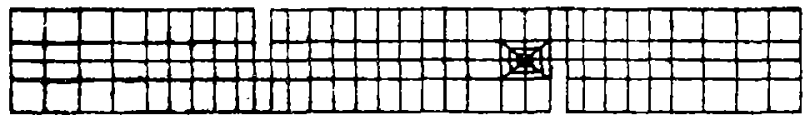

Fig.11 Finite element mesh pattern (II)

There are 24 special elements patched at the near crack tip area. The midside nodes of these elemerits are located according to formula (2.16). The eight crack tip elements are collapsed triangular 
quarter-point singular eiements. On the crack line, all the nodes are multi-displaced nodes. We use the constraint release technique, compute and plot the graphs showing the crack propagation process, Fig. 12 a through Fig.12e.

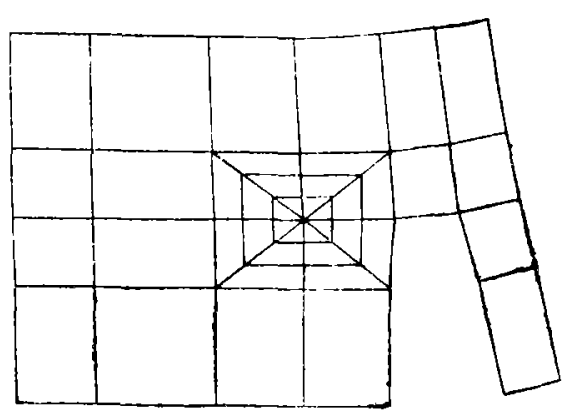

Fig.12a

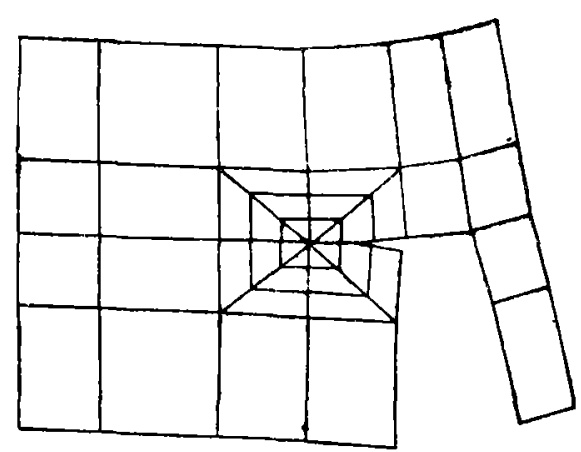

Fig.12c

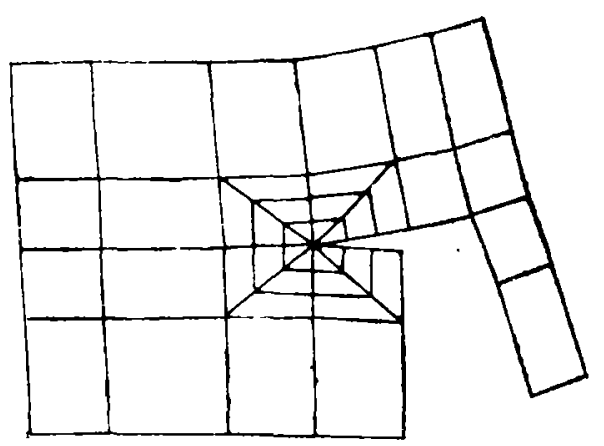

Fig.12e

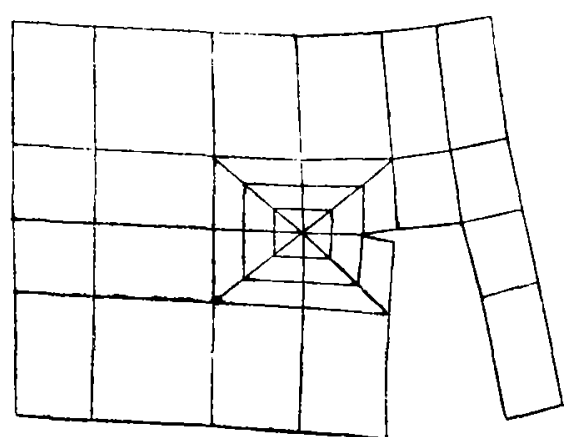

Fig. 12b

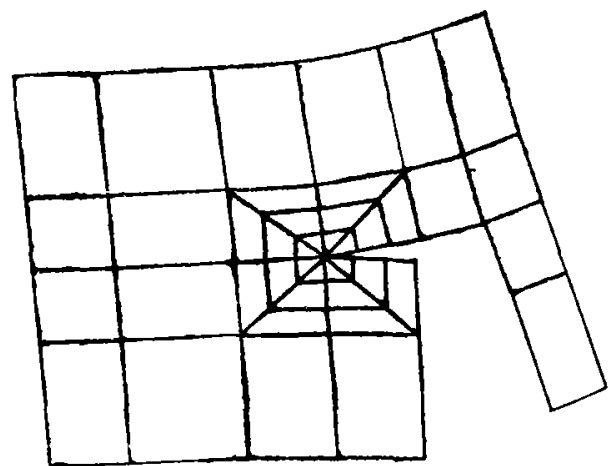

Fig. 12d

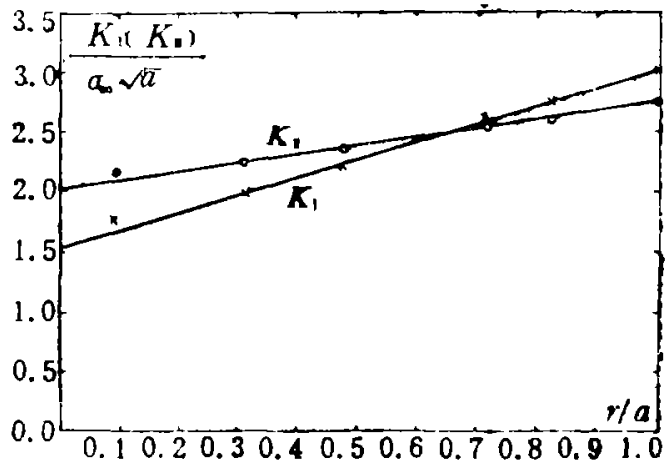

Fig.13 Extrapolation method for defermining $K_{r}$ and $K_{I I}$

The stress intensity factors $K_{I}$ and $K_{I I}$ are computed for both isotropic and orthotropic models. Fig 13 shows the graph of determining $K_{I}$ and $K_{I I}$ from the finite element displacement through extrapolation method. For orthotropic material model, three different approaches to the determination of $K_{I}$ and $K_{I I}$ from displacement are used, the results are summarised in Table 2. The two different cases with and without imposing the constraint condition (3.10) on the collapsed 
three nodes of the crack tip elements are also compared in this Table. The maximum difference between the two cases is for $K_{I I}$ calculated from formula (3.9), amounting to $8.2 \%$ which is not ignorable.

Table 2. A summary of results of $K_{I}$ and $K_{I I}$

\begin{tabular}{|c|c|c|c|}
\hline \multirow{2}{*}{ METHODS } & \multirow{2}{*}{ CONSTRAINT CONDITION } & \multicolumn{2}{|c|}{ STRESS INTENSITY FACTORS } \\
\hline & & $K_{1} /(a-\sqrt[N]{a})$ & $K_{I} /\left(\sigma_{\infty} N_{0}\right)$ \\
\hline \multirow{2}{*}{1 Use Displ. of Point $r_{0}=a / 9$} & Constrained & 1.782 & 2.371 \\
\hline & Free & 1.779 & 2.461 \\
\hline \multirow{2}{*}{2 Formulas (3.9) } & Constrained & 1.383 & 2.285 \\
\hline & Free & 1.386 & 2.488 \\
\hline 3 Extrapolation & Constrained & 1.56 & 2.00 \\
\hline
\end{tabular}

From the three methods of determining $K_{l}$ and $K_{l l}$ from displacement, the maximum difference comes from formula (3.9) and the extrapolation method. The formulas (3.9) are derived from the formulation of the special crack tip element and can produce more accurate results. There is somewhat uncertainty in extrapolation method, the accuracy of this method being questionable. As for the other method, using a single displacement of the point $r_{o}=\mathrm{a} / 9$, to arrive at stress intensity factors $K_{l}$ and $K_{l l}$, the invalidity of its results is obvious.

Finally, the critical stress intensity factors $K_{I C}$ and $K_{I I C}$ are estimated through the consideration of failure load and critical crack length measured in experiment. The maximum tensile stress at remote end is $20 \mathrm{MPa}$, and the critical crack length measured just before the specimen break is approximately $6.5-7 \mathrm{~mm}$. Then from $K_{I}$ and $K_{I I}$ calculated, the estimation of $K_{I C}$ and $K_{I I C}$ for this kind of loading situation are listed in Table 3. These results are comparable with the results given in Ref [8].

Table 3. Critical stress intensity factors

\begin{tabular}{|c|c|c|c|}
\hline & & ISOTROPICMODEL & ORTHOTROPIC MODEL \\
\hline \multirow{2}{*}{$\begin{array}{l}\text { Stress Intensity } \\
\text { Factors }\end{array}$} & $K_{\mathrm{I}} /\left(\sigma_{\infty} \sqrt{a}\right)$ & 1.27 & 1.383 \\
\hline & $K_{x} /\left(\sigma_{\infty} \sim a\right)$ & 2.80 & 2.285 \\
\hline \multirow{2}{*}{$\begin{array}{l}\text { Critical Stress } \\
\text { Intensity } \\
\text { Factors }\end{array}$} & $K_{I_{c}}$ & $66.4 \mathrm{~N} / \mathrm{mm}^{3}$ & $72.3 \mathrm{~N} / \mathrm{mm}^{3}$ \\
\hline & $K_{\mathbf{I}}$ & $146.3 \mathrm{~N} / \mathrm{mm}^{12}$ & $119.4 \mathrm{~N} / \mathrm{mm}$ \\
\hline
\end{tabular}

NB $K_{1}, K_{I I}$, calculated from $(2 b a, b)$

\section{Conclusions}

1 The mixed-mode fracture problems is analysed with FEM. Two kinds of material models (isotropic and homogeneous) are assumed. Both models are homogeneous and the effect of nonhomogeneity is ignored.

2 The stress distributions along shear surface possess a large concentration at the corner of notch, 
this explains why the shear strength obtained by lap-shear test depends on the length of shearsurface.

3 The constraint release technique is utilized for the crack extension process, it can also be used in analsysis of dynamic cracking problems.

4 Three approaches to determination of $K_{I}$ and $K_{I I}$ from finite element displacement are compared. The deviation is not negligibly small, if the division of grids is not very fine. The constraint imposed on the three collapsed nodes renders non-ignorable influence on the displacement very near crack tip. The best way of obtaining linear elastic stress intensity factors is to use the displacement field obtained with the constraint conditions (3.10) and to use formulas (3.9).

\section{References}

[1] Tracy, D. M., Finite elements for determination of crack tip elastic stress intensity factors, Eng. Frac. Mech., 3 (1971), 255-265.

[ 2 ] Henshell, R. D. and K. G. Shaw, Crack tip finite elements are unnecessary, IJNME, 9, 3 (1975). $495-507$.

[ 3 ] Pu, S. L., M. A. Hussain, and W. E. Lorensen, The collapsed cubic isoparametric element as a singular element for crack problems, IJNME, 12, 11, (1978), 1727-1742.

[4] Wang Ke-jen, Hsu Chi-lin and Kao Hua, Calculation of stress intensity factors for combined mode bend specimens, Advances in Research on Strength and Fracture of Materials, 4 (1978), 123.

[ 5] Shih, C. F., H. G. De Lorenzi and M. D. German, Crack extension modelling with singular quadratic isoparametric elements, Int. J. of Fracture, 12 (1976). 647-651.

[6] Barsoum, R. S., Triangular quarter-point elements as elastic and perfectly-plastic crack tip elements, IJNME, 11 (1977), 85-98.

[ 7] Yamada. Y., Y. Ezawa and I. Nishiguchi, Reconsiderations on singularity of crack tip elements, IJNME, 14, 10 (1979), 1525-1544.

[8] Sih, G. C., Fracture mechanics of composite materials, Proceedings of First USA-USSR Symposium on Fracture of Composite Materials, Editors G. C. Sih and V.P. Tamuzs, held in Riga, USSR, 4-7 Sep. (1978), $111-130$.

[9] Wang, S. S., J. F. Yau and H. T. Corten, A mixed-mode crack analysis of rectilinear anisotropic solids using conservation laws of elasticity; Inter. J. Fracture, 16, 3, June (1980), $247-259$.

[10] Owen, M. J., Biaxial failure of GRP-mechanisms, modes and theories, Composite structures 2: Proceedings of the 2nd International Conferences on Composite Structures, held at Paisley College of Technology, Scotland, 14-16, Sep. (1983), Applied Science Publishers (1983). $21-39$. 